\title{
Physical meaning of the equinoctial effect for semi-annual variation in geomagnetic activity
}

\author{
A. Yoshida \\ National Institute of Polar Research, 1-9-10 Kaga, Itabashi, Tokyo, 173-8515, Japan \\ Received: 15 September 2008 - Revised: 6 February 2009 - Accepted: 7 April 2009 - Published: 4 May 2009
}

\begin{abstract}
Physical meaning of the equinoctial effect for semi-annual variation in geomagnetic activity is investigated based on the three-hourly am index and solar wind parameters. When the $\mathrm{z}$ component of the interplanetary magnetic field (IMF) in geocentric solar magnetospheric (GSM) coordinates is southward, am indices are well correlated with $B_{s} V_{x}^{2}$, where $B_{s}$ is the southward component of the IMF and $V_{x}$ is the solar wind velocity in the sun-earth direction. The $a m-B_{s} V_{x}^{2}$ relationship, however, depends on the range of $V_{x}^{2}$ : the $a m$ in higher ranges of $V_{x}^{2}$ tends to be larger than $a m$ in lower ranges of $V_{x}^{2}$ for the same value of $B_{s} V_{x}^{2}$ for both equinoctial and solstitial epochs. Using the data sets of the same $V_{x}^{2}$ range, it is shown that distribution of points in the $a m-B_{s} V_{x}^{2}$ diagram at the solstitial epochs overlaps with that at the equinoctial epochs and the average $a m$ values in each $B_{s} V_{x}^{2}$ bin in solstitial epochs are closely consistent with those in equinoctial epochs, if $V_{x}^{2}$ for each point at solstices are reduced to $V_{x}^{2} \sin ^{2}(\Psi)$ where $\Psi$ is the geomagnetic colatitude of the sub-solar point. Further, it is shown that monthly averages of the am index in the long period is well correlated with the values of $\sin ^{2}(\psi)$ for the middle day of each month. These findings indicate that the factor that contributes to the generation of geomagnetic disturbance is not the velocity of the solar wind, but the component of the solar wind velocity perpendicular to the dipole axis of the geomagnetic field. The magnitude of the perpendicular velocity component varies semi-annually even if the solar wind velocity remains constant, which is considered to be the long-missed key factor causing the equinoctial effect.
\end{abstract}

Keywords. Magnetospheric physics (Solar windmagnetosphere interactions)

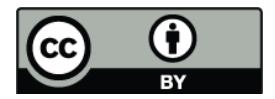

Correspondence to: A. Yoshida (ayoshida@nipr.ac.jp)

\section{Introduction}

The general tendency for magnetic disturbances to be more stormy at equinoxes than at solstices has been recognised for more than 150 years (Sabine, 1852) and the cause of the semi-annual variation has been studied by many researchers since them. Three principal hypotheses have been proposed; the axial hypothesis (Cortie, 1912), the equinoctial hypothesis (Bartels, 1932; McIntosh, 1959), and the Russell and McPherron (RM) hypothesis (Russell and McPherron, 1973). The axial hypothesis considers the heliographic latitude of the earth, relating the enhanced geomagnetic activity in March and September to the fact that the sub-earth point is at that time most separated from the solar equatorial plane and hence closer to active spot regions where eruptive phenomena often occur or nearer to mid-latitude coronal holes from which high-speed wind flows out. The key parameter in the equinoctial hypothesis, on the other hand, is the geomagnetic colatitude $(\Psi)$ of the sub-solar point, that is, the angle between the solar wind flow and the dipole axis of the earth. However, it has not been elucidated why and in what way the geomagnetic colatitude is involved in the semi-annual modulation of geomagnetic activity. The RM hypothesis is based on the recognition that the magnetic field in the solar equatorial plane tends to have the largest southward component in geocentric solar magnetospheric (GSM) coordinates in early April and October, depending on polarity.

The explanation by the RM hypothesis has been generally accepted for many years. This may be primarily because it related the semi-annual variation of geomagnetic activity to the change in the southward component of the IMF which plays an essential role in the dayside magnetic reconnection between the interplanetary magnetic field (IMF) and the geomagnetic field. However, it was argued from very early time after its proposition that the RM effect is not enough to explain the full amplitude (Murayama, 1974; Berthelier, 1976; Schreiber, 1981). It has also been pointed out that the diurnal

Published by Copernicus Publications on behalf of the European Geosciences Union. 
(a)

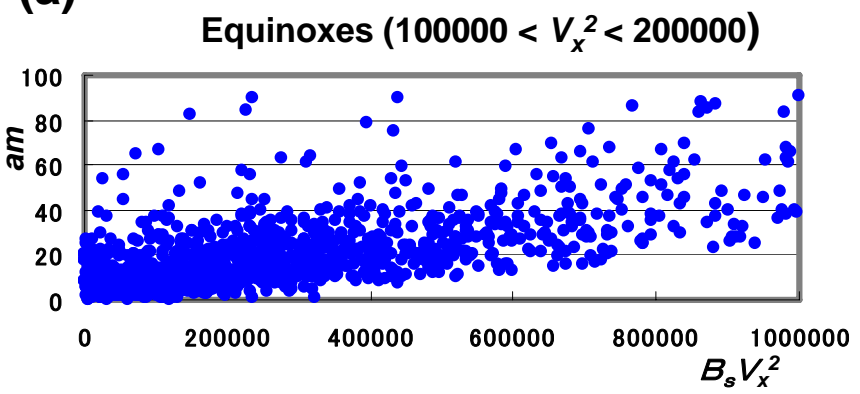

Equinoxes $\left(200000<V_{x}^{2}<300000\right)$

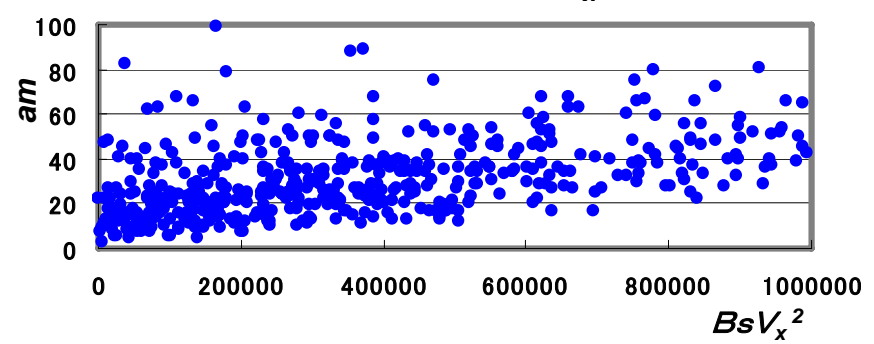

Equinoxes $\left(300000<V_{x}^{2}<400000\right)$

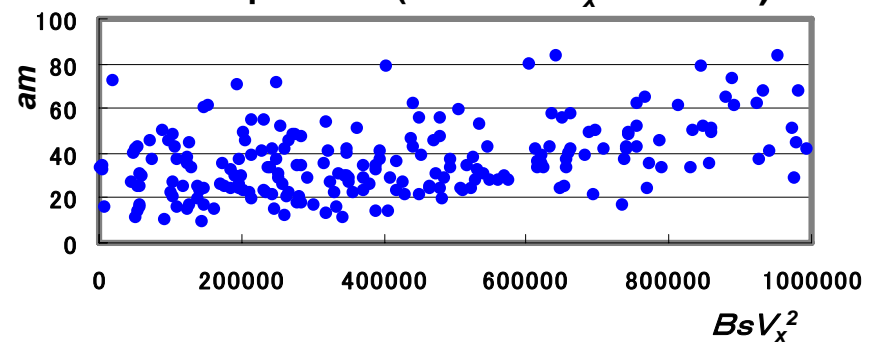

(b)
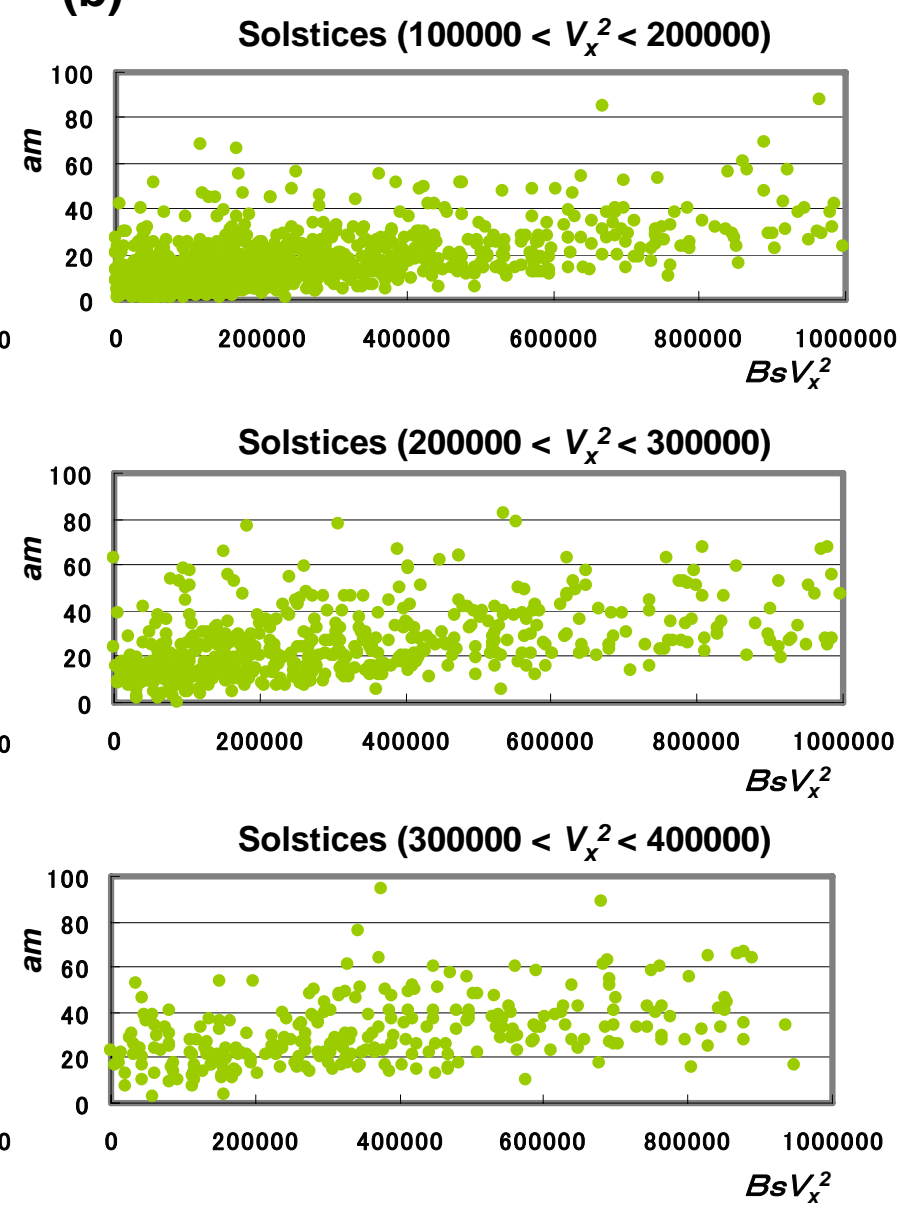

Fig. 1. Plot of geomagnetic am index versus $B_{S} V_{x}^{2}$ (GSM) for 1998-2007 in velocity ranges of (upper) $100000<V_{x}^{2}<200000$, (middle) $200000<V_{x}^{2}<300000$, and (lower) $300000<V_{x}^{2}<400000$, where $V_{x}$ is the solar wind velocity in the sun-earth direction in $\mathrm{km} / \mathrm{s}$ and $B_{S}$ is the magnitude of the southward component of the IMF in nT. (a) Equinoctial epochs ( \pm 15 days from equinoxes). (b) Solstitial epochs ( \pm 15 days from solstices).

variation does not accord with the characteristics predicted by the RM hypothesis (Mayaud, 1978; Berthelier, 1990). A lot of studies have been done to clarify what a portion of the semi-annual variation in geomagnetic activity is attributed to each of the mechanisms (Cliver et al., 2000, 2002, 2001; O'Brien and McPherron, 2002). de La Sayette and Berthelier (1996) pointed out another effect which would arise from the feature that $B_{z}$ in the geocentric solar equatorial (GSEQ) coordinate system has $B_{z}$ component in the GSM coordinates. By the elaborate analysis, they showed that a characteristic pattern in the annual-diurnal variations derived from the effect coincides with the pattern appearing in the part of the geomagnetic activity which is not dependent on the IMF polarity.

It is very likely that various effects as described above work together to produce the annual-diurnal variations in geomagnetic activity. The purpose of the present study is not to evaluate contribution of each effect to the phenomena, but to investigate physical meaning of the equinoctial effect. Recent detailed studies based on am and $D_{s t}$ indices have shown that the equinoctial effect plays the dominant role in the semi-annual variation in geomagnetic activity (Cliver et al., 2000, 2001; O'Brien and McPherron, 2002), but yet, the physical mechanism by which the equinoctial effect affects geomagnetic activity has not been clarified (Cliver et al., 2004). The present study proposes an idea how the equinoctial effect works.

\section{Data and analysis}

We investigate the semi-annual variation observed in the am index. Mid-latitude range indices, such as $a m$, have been shown in many studies to be closely correlated with the product of $B_{s} V_{x}^{2}$, where $B_{s}$ is the southward component of the IMF in GSM coordinates, and $V_{x}$ is the solar wind velocity 
in the sun-earth direction (Svalgaard, 1977; Feynman, 1980; Maezawa and Murayama, 1986), although the coefficients appearing in the linear relation between $a m$ and $B_{s} V_{x}^{2}$ differ somewhat according to the data period and averaging procedures used to derive the relationship. In the present study, the relation between am index calculated at an interval of $3 \mathrm{~h}$ (Menvielle and Berthelier, 1991) and the solar wind parameters is investigated directly. The values for the solar wind velocity and the IMF were taken from the Level 2 hourly data acquired by the Advanced Composition Explorer (ACE) satellite. The delay between passage of the solar wind past the ACE satellite and the time at which the wind encounters the earth (ca. $1 \mathrm{~h}$ ) is accounted for by shifting the hourly $B_{S}$ and $V_{x}$ data by $1 \mathrm{~h}$ to afford a data set of three-hourly mean values for $B_{s}$ and $V_{x}$ corresponding to the $a m$ index.

Figure $1 \mathrm{a}, \mathrm{b}$ plots am against $B_{s} V_{x}^{2}$ for equinoctial and solstitial epochs ( \pm 15 days from equinoxes and solstices) for the period 1998-2007. As the $a m-B_{s} V_{x}^{2}$ relationship derived from the three-hourly data set was found to be dependent on the range of $V_{x}^{2}$ (Yoshida, 2009a), the am and solar wind data were divided into separate data sets according to the range of $V_{x}^{2}$, and the semi-annual variation in each data set was investigated separately. Figure 1 shows the $a m$ versus $B_{s} V_{x}^{2}$ relationship for three ranges of $V_{x}^{2}$. When diagrams for the data sets of the same $V_{x}^{2}$ ranges are compared, a tendency is seen that the am value is larger at equinoxes than at solstices for the same value of $B_{s} V_{x}^{2}$ in average, illustrating the equinoctial effect. However, it is also seen from the plots in Fig. 1 that $a m$ in higher ranges of $V_{x}^{2}$ is obviously larger than $a m$ in lower ranges of $V_{x}^{2}$ for the same $B s V_{x}^{2}$ values for both equinoctial and solstitial epochs. Owing to this feature, if average of $V_{x}^{2}$ at solstitial epochs is larger than average of $V_{x}^{2}$ at equinoctial epochs, we would obtain an unexpected result that the average $\mathrm{am}$ at solstices is larger than the average $\mathrm{am}$ at equinoxes. It is therefore of critical importance in the investigation of hidden factor in the equinoctial effect to compare the $a m-B_{s} V_{x}^{2}$ relationships for equinoctial and solstitial epochs based on data sets of the same range of $V_{x}^{2}$.

The dependence of the $a m-B_{s} V_{x}^{2}$ relationship on the range of $V_{x}$ does not mean that am should be more appropriately scaled according to a higher power of $V_{x}$. It was confirmed that a power $(n)$ greater than 2 does not change the situation. That is, the $a m-B_{s} V_{x}^{n}$ relationship for which $n$ is greater than 2 still depends on the range of $V_{x}$ : The larger the range of $V_{x}$, the larger the $a m$ for the same value of $B_{s} V_{x}^{n}$ (Yoshida, 2009a). This dependence of the $a m-B_{s} V_{x}^{2}$ relationship on the range of $V_{x}$ is very likely to have some significant physical meaning in relation to the efficiency of the dayside magnetic reconnection. However, a detailed examination of such a meaning is beyond the scope of the present study. We only briefly note here that the dependence of the $a m-B_{s} V_{x}^{2}$ relationship on the range of $V_{x}$ is considered to indicate that the efficiency of the merging of magnetic fields depends on not only the influx of $B_{S}$ of the IMF, but also the configuration of the magnetopause which is basically determined by the balance between the Earth's magnetospheric magnetic pressure and the pressure of bombarding solar wind particles. This feature may be related to the variation of the strength of magnetic field reconnection with the depth to which the solar wind penetrates into the geomagnetic field (Crooker and Siscoe, 1986).

Previous studies investigating the physical meaning of the equinoctial effect postulated that the equinoctial effect is independent of solar wind parameters, and that the influences of each on geomagnetic activity are separable (Svalgaard, 1977, 2002; Cliver et al., 2004). It was found in the present study, however, that the rate of reduction of average $\mathrm{am}$ at solstices apparently differs for different values of $B_{s} V_{x}^{2}$, if all the data are included in the calculation. This finding as well as the recognition of the features observed in Fig. 1 led the author to think that the key parameter relevant to the equinoctial effect may be hidden in the solar wind velocity. The idea that the solar wind velocity effective to the emergence of geomagnetic disturbance might be its component perpendicular to the dipole axis was conceived intuitively, not logically. But it was immediately understood that the idea readily explains why the colatitude $\Psi$ of the sub-solar point plays an essential role in the equinoctial effect, for the perpendicular component of the solar wind velocity is obtained by multiplying the factor $\sin (\Psi)$. A crucial point of this idea is that the perpendicular component changes semi-annually even if the solar wind velocity remains constant throughout the year.

The proposed idea is examined quantitatively in Fig. 2. In Fig. 2a, b, am index is plotted against $B_{s} V_{x}^{2}$ for equinoctial and solstitial epochs, respectively. The range of $V_{x}^{2}$ in these diagrams is constrained to $100000<V_{x}^{2}<200000$ in order to ensure valid data comparison, and according to the idea, observed $V_{x}^{2}$ values at solstitial epochs are reduced by a factor of 0.841 , corresponding to $\sin ^{2}(66.5)$, where 66.5 is the average angle of the solar wind flow direction to the dipole axis of the earth at solstices. Multiplication by this factor thus affords the solar wind velocity component perpendicular to the dipole axis of the earth. The resultant distribution of points for solstitial epochs overlaps the data for equinoctial epochs excellently (Fig. 2c). Figure 3 shows that the average am values in each $B_{s} V_{x}^{2}$ bin in solstitial epochs, where $V_{x}^{2}$ is reduced by a factor 0.841 , are closely consistent with those in equinoctial epochs. This result confirms that the component of solar wind velocity that is effective in producing geomagnetic disturbance in mid latitude is that perpendicular to the dipole axis of the earth. This component is considered to be the long-missed physical factor giving rise to the equinoctial effect.

\section{Monthly averages of the am index}

The semi-annal variation in geomagnetic activity is not discernable in the monthly averages of the am index over short intervals due to the relative dominance of the effect of solar 


\section{(a)}
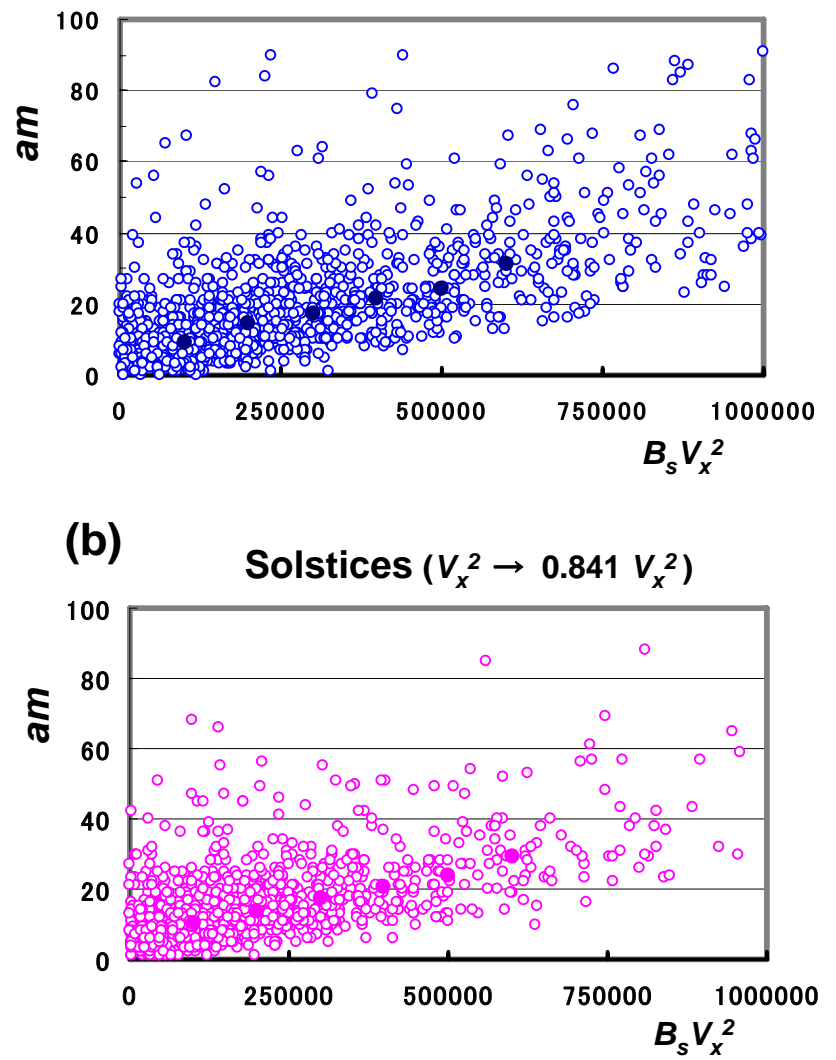

\section{(c)}

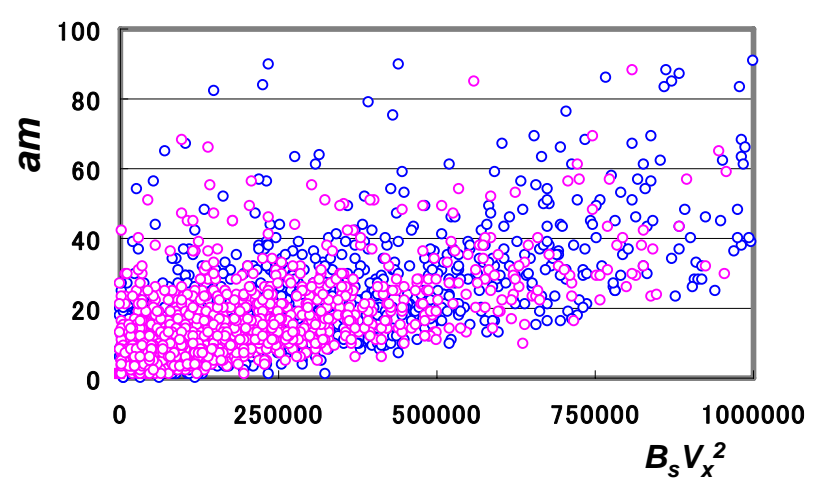

Fig. 2. $a m$ index versus $B_{S} V_{x}^{2}$ at equinoxes (a), and at solstices (b), for $100000<V_{x}^{2}<200000$, where $V_{x}$ is reduced by a factor sin (66.5) for solstices. Average values for several $B_{S} V_{x}^{2}$ bins are shown by large blue and red circles, respectively. (c) Superposition of the $a m-B_{S} V_{x}^{2}$ diagrams for equinoxes (blue) and solstices (red).

wind velocity variation. Monthly mean values of the am index increase according to the square of the mean velocity, and this behaviour obscures the equinoctial effect on the variation in the am index. When the mean value of $V_{x}^{2}$ at solstices is larger than that at equinoxes, the mean am index at solstices could also be larger than that at equinoxes (see Fig. 1).

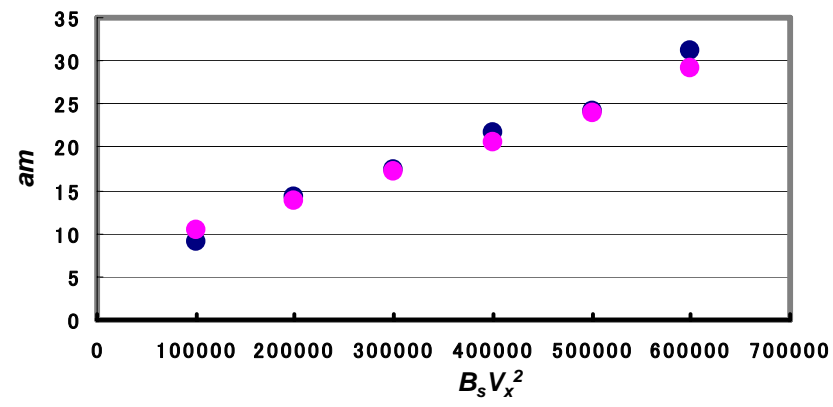

Fig. 3. Comparison of average $a m$ values for each $B_{S} V_{x}^{2}$ bin for equinoxes (blue circles) and those for solstices (red circles). Solar wind velocity is reduced by a factor $\sin (66.5)$ for solstices.

However, if the am index is analyzed over a sufficiently long period, the monthly averages exhibit a distinct semi-annual variation. Figure 4a shows the semi-annual variation in the monthly averages of the am index over a 47-year period from 1961 to 2007 . Over such a long period, the average and dispersion of solar wind velocity may become essentially uniform for all months. The monthly averages of the am index are plotted against $\sin ^{2}(. \psi)$ in Fig. $4 b$, where values of $\sin ^{2}(\psi)$ are calculated for the middle day of each month. It is seen that the two values are well correlated, representing further evidence in support of the proposal that the equinoctial effect is caused by the semi-annual change in $\sin ^{2}(\psi)$.

An empirical function including a variable $\cos ^{2}(\psi)$ has been previously proposed to explain the semi-annual variation in the average am index (Svalgaard, 1977, 2002). We confirmed that the empirical function is also well correlated with the monthly averages of the am index. Interestingly, there is a good correlation between $\sin ^{2}(\psi)$ and the empirical function, as shown in Fig. 4c. This is not surprising, since the first order of the Taylor expansion of the empirical function reduces to a linear function of $\sin ^{2}(\psi)$. The physical meaning of the empirical function, however, is not clear (Svalgaard, 2002). On the other hand, the function $\sin ^{2}(\psi)$ has a sound physical basis in that it expresses the semi-annual change in the velocity component perpendicular to the dipole axis, which is supposed to be the effective component of solar wind velocity relevant to the solar wind-magnetosphere coupling.

\section{Discussion}

According to the equinoctial hypothesis emergence of geomagnetic disturbance is regulated by the angle between the solar wind flow and the dipole axis of the earth $(\psi)$, with the weakest geomagnetic activity at solstices when $\psi$ reaches a minimum. The Russell-McPherron (RM) hypothesis, on the other hand, asserts that the solar wind-magnetosphere coupling is most enhanced at equinoxes, because $B_{s}$ tends to take maximum values at the epochs. The RM effect has 
been regarded as a most satisfactory explanation for the semiannual variation in geomagnetic activity. This is primarily because the RM effect is closely related to the magnetic reconnection between the interplanetary magnetic field (IMF) and the geomagnetic field, which involves the southward component of the IMF as a principal part. It was pointed out that characteristics of the diurnal variation does not accord with the prediction by the RM hypothesis, but it coincides with the prediction by the equinoctial hypothesis (Mayaud, 1978; Berthelier, 1990), nevertheless, the equinoctial hypothesis has been disfavoured due to the absence of a mechanism that relates the effect to the magnetic merging process. Our finding affords the long-sought explanation that relates the equinoctial effect to the magnetic merging process: Geomagnetic activity at solstices becomes weak because the solar wind velocity effective to the magnetic reconnection is reduced by a factor $\sin ^{2}(\Psi)$, where $\Psi$ is the angle between the solar wind flow direction and the dipole axis of the earth at solstices. If this explanation is valid, it raises an old problem about the site of the dayside magnetic merging, for it seems to suggest that the magnetic reconnection is likely to occur near the magnetic equator of the earth rather than at the sub-solar point where the solar wind collides on the magnetopause. There have been a lot of arguments about the site of reconnection. This is an important problem closely related to the mechanism by which the solar wind energy enters into the magnetosphere. However, the thorough discussion on the problem is beyond the scope of this paper. We will treat this issue from wider points of view in another paper where emergence of high- and mid-latitudes geomagnetic disturbances is discussed in relation to the magnetic merging at the dayside magnetopause (Yoshida, 2009b).

The explanation for the cause of the equinoctial effect proposed in the present study is surprisingly simple, but we think it solves a problem that has remained unsolved for many years. Such a simple explanation of the equinoctial effect may have been overlooked in previous studies for two reasons. The first reason is that the semi-annual variation recognized in the average values of the am index or $a a$ index in long periods is used as a basis of consideration in most of previous analyses, whereas the am indices are directly compared to the three-hourly values of solar wind parameters in the present study. Studies on average values of the am index in a long period are likely to miss the essential feature of the equinoctial effect, that is, that the effective solar wind velocity related to the generation of magnetic disturbance changes according to $\sin (\psi)$. The second reason is the classification of the data sets according to the ranges of $V_{x}^{2}$, which is particularly important in the quantitative evaluation of the equinoctial effect. Although it was reported that the mean am index at equinoxes tends to be larger than that at solstices for the same value of $B_{s} V_{x}^{2}$, (Cliver et al., 2000), the need to constrain data to sets of the same $V_{x}^{2}$ ranges in order to evaluate the equinoctial effect quantitatively has been overlooked. (a)

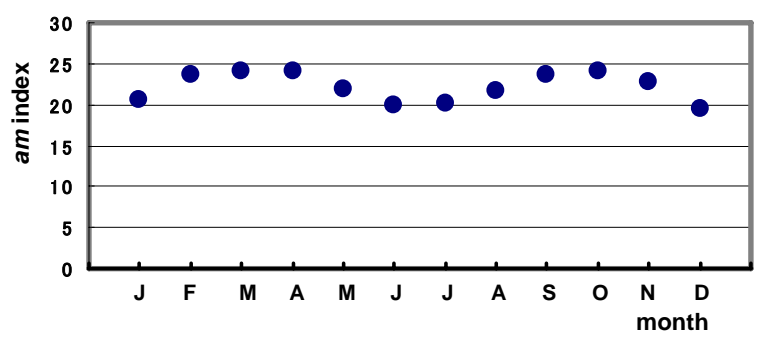

(b)

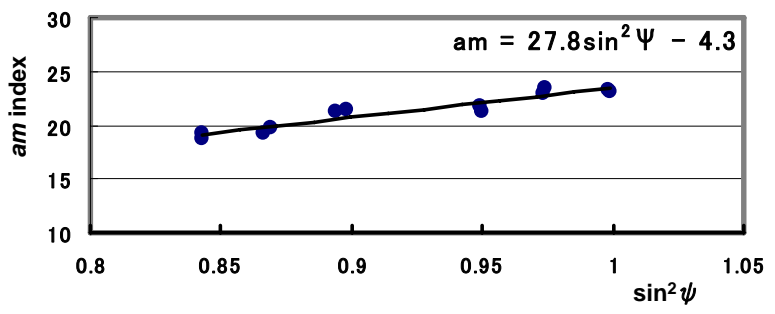

(c)

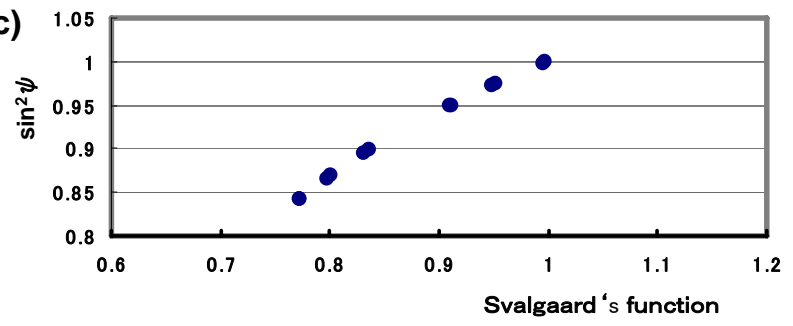

Fig. 4. Relationship between monthly averages of am index and $\sin ^{2}(\psi)$. (a) Semi-annual variation in monthly am averages for the period 1961-2007. (b) Monthly average of the am indices versus $\sin ^{2}(\psi)$ at the middle day of each month. (c) Comparison of $\sin ^{2}(\psi)$ with Svalgaard's empirical function.

The equinoctial effect was once explained as being related to the Kelvin-Helmholtz instability at the boundary of the magnetosphere (Boller and Stolov, 1975). However, it was soon shown that the model requires more energy should be transferred by the Kelvin-Helmholtz waves than by magnetic merging, which is very unlikely (Hill, 1979). This realisation prompted researchers to search for a mechanism that connects the equinoctial effect to the merging process in which $B_{s}$ plays an essential role, although there was no clue to relate the equinoctial effect to $B_{s}$. The explanation proposed in the present study shows that the physical basis was not in the phenomena related to $B_{s}$, but in the velocity component that is involved as another principal factor in the magnetic reconnection process. It is interesting to note that both the $\mathrm{RM}$ and equinoctial effects are related to seasonal changes in the efficiency of solar wind-magnetosphere coupling caused by changes in the geometric configuration between the sun and the geomagnetic dipole field, one in relation to $B_{S}$ of the IMF, and the other in relation to the component of solar wind velocity perpendicular to the dipole axis. 
Acknowledgements. The am index data were provided by the International Service of Geomagnetic Indices (ISGI). Gratitude is extended to the ACE SWEPAM instrument team and the ACE Science Center for providing ACE data. The author thanks L. Svalgaard and two anonymous referees for their valuable comments, and $\mathrm{M}$. $\mathrm{Ku}-$ nitake and K. Tanikawa for helpful discussions.

Topical Editor I. A. Daglis thanks two anonymous referees for their help in evaluating this paper.

\section{References}

Bartels, J.: Terrestrial-magnetic activity and its relation to solar phenomena, Terr. Mag. Atmos. Electr., 37, 1-52, 1932.

Berthelier, A.: Influence of the polarity of the interplanetary magnetic field on the annual and diurnal variations of magnetic activity, J. Geophys. Res., 81, 4546-4551, 1976.

Berthelier, A.: Comments on "The universal time variation of magnetic activity," Geophys. Res. Lett., 17, 307-308, 1990.

Boller, B. R. and Stolov, H.: Kelvin-Helmholtz instability and the semiannual variation of geomagnetic activity, J. Geophys. Res., 75, 6073-6084, 1975.

Cliver, E. W., Kamide, Y., and Ling, A. G.: Mountains versus valleys: Semiannual variation of geomagnetic activity, J. Geophy. Res., 105, 2413-2424, 2000.

Cliver, E. W., Kamide, Y., and Ling, A. G.: The semiannual variation of geomagnetic activity: phases and profiles for 130 years of a data, J. Atmos. Solar-Terr. Phys., 64, 47-53, 2002.

Cliver, E. W., Kamide, Y., Ling, A. G., and Yokoyama, N.: Semiannual variation of the geomagnetic $D_{s t}$ index: Evidence for a dominant nonstorm component, J. Geophy. Res., 106, 2129721304, 2001.

Cliver, E. W., Svalgaard, L., and Ling, A. G.: Origins of the semiannual variation of geomagnetic activity in 1954 and 1996, Ann. Geophys., 22, 93-100, 2004, http://www.ann-geophys.net/22/93/2004/.

Cortie, A. L.: Sunspots and terrestrial magnetic phenomena, 18981911, Mon. Notic. R. Astron. Soc., 73, 52-60, 1912.

Crooker, N. U. and Siscoe, G. L.: On the limits of energy transfer through dayside merging, J. Geophys. Res., 91, 13393-13397, 1986. de La Sayette, P. and Berthelier, A.: The am annual-diurnal variations 1959-1988: A 30-year evaluation, J. Geophys. Res., 10653-10663, 1996.

Feynman, J.: Implications of solar cycles 19 and 20, Geophys. Res. Lett., 7, 971-973, 1980.

Hill, T. W.: Rates of mass, momentum, and energy transfer at the magnetopause, Magnetospheric Boundary Layers, edited by Battrick, B, Eur. Space Agency Spec. Publ., ESA SP-148, 325-332, 1979.

Maezawa, K. and Murayama, T.: Solar wind velocity effects on the auroral zone magnetic disturbances, in: Solar WindMagnetosphere Coupling, edited by: Kamide Y. and Slavin, J. A., pp. 59-83, Terra Scientific, Tokyo, 1986.

Mayaud, P. N.: The annual and daily variations of the $D s t$ index, Geophys. J. R. Astron. Soc., 55, 193-201, 1978.

McIntosh, D. H.: On the annual variation of magnetic disturbance, Phil. Trans. Roy. Soc. London, Ser. A, 251, 525-552, 1959.

Menvielle, M. and Berthelier, A.: The K-derived planetary indices: Description and availability, Rev. Geophys., 29, 415-432, 1991.

Murayama, T.: Origin of the semiannual variation of geomagnetic Kp indices, J. Geophys. Res., 79, 297-300, 1974.

O'Brien, T. P. and McPherron, R. L.: Semiannual and diurnal variation of Dst dynamics, J. Geophys. Res., 107(A11), 1341, doi:10.1029/2002JA009435, 2002.

Russell, C. T. and McPherron, R. L.: Semiannual variation of geomagnetic activity, J. Geophys. Res., 78, 92-108, 1973.

Sabine, E.: On periodical laws discoverable in the mean effects of the larger magnetic disturbances, II, Philos. Trans. R. Soc. London, 142, 103-124, 1852.

Schreiber, H.: Correlation of geomagnetic activity indices Ap with the solar wind speed and southward interplanetary magnetic field, J. Geophys., 49, 169-175, 1981.

Svalgaard, L.: Geomagnetic activity: Dependence on solar wind parameters, in: Coronal Holes and High Speed Wind Streams, edited by: Zirker, J. B., pp. 371-441, Colo. Assoc. Univ. press, Boulder, Colo., 1977.

Svalgaard, L.: The semiannual variation of great geomagnetic storms, Geophys. Res. Lett., 29(16), 12, doi:10.1029/2001GL014145, 2002.

Yoshida, A.: Why was the geomagnetic activity in 2003 extraordinarily high?, J. Atmos. Solar-Terr. Phys., in review, 2009a.

Yoshida, A.: Semi-annual variations in the $A E$ and $a m$ indices, Japan Geoscience Union Meeting 2009, E118, 2009 b. 